\title{
EchoGéo
}

2 | 2007

Itinéraires et trajectoires des migrants

\section{Vidéo et pratique de la Géographie}

\author{
Gaëlle Hallair
}

\section{OpenEdition}

Journals

Édition électronique

URL : https://journals.openedition.org/echogeo/1643

DOI : $10.4000 /$ echogeo.1643

ISSN : 1963-1197

\section{Éditeur}

Pôle de recherche pour l'organisation et la diffusion de l'information géographique (CNRS UMR 8586)

\section{Référence électronique}

Gaëlle Hallair, «Vidéo et pratique de la Géographie », EchoGéo [En ligne], 2 | 2007, mis en ligne le 13 mars 2008, consulté le 31 juillet 2021. URL : http://journals.openedition.org/echogeo/1643 ; DOI : https://doi.org/10.4000/echogeo.1643

Ce document a été généré automatiquement le 31 juillet 2021.

EchoGéo est mis à disposition selon les termes de la licence Creative Commons Attribution - Pas d'Utilisation Commerciale - Pas de Modification 4.0 International (CC BY-NC-ND) 


\title{
Vidéo et pratique de la Géographie
}

\author{
Gaëlle Hallair
}

« Penser l'image et penser en images, c'est aussi placer le géographe du côté du vivant ${ }^{1} »$.

1 L'ambition affichée est d'intégrer la vidéo et la pratique de l'audiovisuel dans la recherche en géographie. Les géographes utilisent depuis longtemps l'image: le magnifique fonds de photographies de plaques de verre ${ }^{2}$ constitué par des géographes comme Emmanuel de Martonne, Jean Dresch ou Charles Robequain l'atteste. Si l'audiovisuel occupe une place importante chez les ethnologues (l'un des pionniers étant Jean Rouch), il reste encore marginal chez les géographes. Citons cependant quelques noms de géographes-vidéastes : Philippe Pinchemel, Jean-Louis Tissier, Claude Collin-Delavaud, Lucien Faugères, Luc Federmeyer, Jean-Loïc Portron, Xavier Browaeys, Olivier Archambault. Pourquoi une telle timidité des géographes face à l'audiovisuel? Les blocages sont beaucoup moins techniques que l'on croit; ils résident surtout dans les esprits. A l'ère du numérique et de la miniaturisation, l'utilisation d'une caméra n'est pas plus compliquée que celle d'un appareil photographique. La rigueur demandée dans la pratique de l'audiovisuel est semblable à celle requise pour toute démarche scientifique.

2 L'expérience audiovisuelle est effective depuis plusieurs années à l'UMR PRODIG. Si quelques chercheurs comme Jérôme Fournier utilisent la vidéo comme outil de recherche pour enregistrer les évolutions littorales, la plupart ont pour objectif de valoriser leurs recherches de terrain par un film documentaire. Par ailleurs, les liens institutionnels avec l'université de Paris1 Panthéon Sorbonne et notamment l'atelier Géo-vidéo de Tolbiac permettent à des étudiants de Master et de thèse de réaliser ce qui constitue souvent leur premier film.

1. Inventaire de quelques films documentaires réalisés et en cours de réalisation

3 Avant 2002: montage sur table de montage U-matic, d'où la nécessité de dupliquer les films en version numérique

- «Tiolté ou la décrue du Djoliba », réalisé par Joël Gaudin (auteur scientifique : Philippe Chamard), produit par CNRS-PRODIG ; CNRS AV ; ORTM; Univ. Paris X, 48 minutes, 1999, (disponible en VHS à CNRS Images ). 
- « Naaré ou la montée des eaux » réalisé par Joël Gaudin, (auteurs scientifiques : Philippe Chamard, Marie-Françoise Courel), produit par CNRS-Imagéo; CNRS AV, format video 8, 37 minutes, 1992, (disponible en VHS à CNRS Images).

A partir de 2002 : montage numérique

- «Venez vous faire visiter ! ", réalisé par Solenne Philippon, 13 minutes, production PRODIG, 2003-2007, (disponible en DVD)

- « Battre et vanner son mil chez les Dii et les Duupa du Nord Cameroun », réalisé par

C. Raimond et E. Garine, 13 minutes, 2002, coproduction PRODIG/Images Média/CNRS, (disponible en DVD)

- «Rive gauche Paris » réalisé par Xavier Browaeys et Aliki Dionnet, 40 minutes, 2006, PRODIG

- «"Toute la mémoire du monde" Sainte-Croix du Verdon » réalisé par Xavier Browaeys et Martine Tabeaud, 42 minutes, 2006.

Les projets audiovisuels en cours sont les suivants

- «Autobio-géographie », réalisé par Jean-Marie Théodat, 52 minutes.

- « Monsieur Lampin », réalisé par Jean-Marie Théodat, 26 minutes.

- «Birmanie, histoire d'un soulèvement manqué », réalisé par Guy. Lubeigt.

- «Birmanie, terre de l'or et des pagode », série de clips vidéo à intégrer dans le projet multimédia du même nom en cours de réalisation.

- «Cervières : histoire d'un village (Loire) » réalisé par Xavier Browaeys et Paul Châtelain.

2. Moyens audiovisuels disponibles à Prodig

6 PRODIG dispose d'une unité de tournage haute définition avec son HF et d'une unité de montage avec les logiciels iMovie et Final Cut Express.

L'expérience audiovisuelle des chercheurs de PRODIG est pour l'instant modeste. Elle s'inscrit dans une démarche totalement différente de celle de CNRS Images. En effet, à PRODIG, les chercheurs sont les réalisateurs.

8 En outre, les aspects techniques sont pris en charge par les 2 ITA de la cellule audiovisuelle.

3. Pratique de la vidéo à Prodig : une partition à quatre mains

9 Le binôme chercheur-monteur constitue l'originalité de la pratique audiovisuelle à Prodig. L'objectif visé est aussi de former les enseignants-chercheurs qui le souhaitent à la réalisation complète et quasi-autonome d'un documentaire.

10 Le tandem chercheur - monteur suit une méthodologie qui se déroule en quatre mouvements.

Le travail sur le synopsis-scénario

11 Ce travail est différent de l'écriture d'un article de recherche, en général plutôt analytique et permettant d'aller très finement dans le détail et la spécialisation. Pour un synopsis, l'écriture est autre : plus concise, plus synthétique, elle doit faire ressortir deux ou trois idées force. Ces idées-phares vont ensuite être déroulée selon un fil rouge dans une logique narrative.

Le synopsis est une courte déclaration d'intention qui vise à présenter le sujet du film, les thèses défendues et les moyens cinématographiques (visuels et sonores) mis en œuvre. Le synopsis permet d'élaborer le scénario. Le scénario, plus précis et organisé en grandes séquences (parties) elles-mêmes divisées en scènes, est la base sur laquelle s'appuiera le tournage. (Browaeys, 1999, p.30). 
Armé du scénario (provisoire), on peut ensuite passer à la prise de vues et de sons sur le terrain de recherche

Quelques consignes simples à respecter (parmi d'autres) :

- Filmer avec un pied sinon beaucoup d'images sont inutilisables au montage

- Soigner particulièrement le son par exemple en utilisant un micro spécifique pour les interviews et des oreillettes de contrôle. Penser à enregistrer des sons pour se constituer une petite banque de sons très utile au montage. Le son est déterminant dans la réussite d'un film. Il donne une ambiance et scelle la différence entre un film amateur et un film plus professionnel

- Soigner l'éclairage (éviter de filmer en contre-jour, faire la balance des blancs, etc.)

- Prévoir des cassettes et des batteries de rechange...

- Protéger la caméra : par exemple avec du film alimentaire si on filme dans des endroits susceptibles d'être secs et poussiéreux (désert, Sahel, route non goudronnée, zone de chauffage au bois, etc.)

Le montage des images

Il s'opère sur un ordinateur mac G5 grâce à l'un des deux logiciels de montage que sont iMovie et Final Cut Express. Le travail se poursuit en binôme; il est le fruit d'une discussion permanente entre le chercheur réalisateur et le monteur: le réalisateur exprime ce qu'il veut démontrer dans son film et le monteur traduit cela en langage vidéo et propose des solutions de montage. Pour des raisons d'accessibilité aux logiciels et de sécurité informatique, c'est le monteur qui manipule le logiciel de montage.

15 Voir illustration en fichier joint

Dans iMovie, le montage des clips vidéo se fait dans la timeline (en bas) tandis que les clips non utilisés restent dans le chutier (à droite en colonne).

Le mixage du son

17 C'est la phase à la fois la plus délicate et peut être la plus captivante. Il est vrai aussi que le mixage du son apporte de façon décisive la touche finale pour l'atmosphère d'un film: par le commentaire en voix off, par les sons d'ambiance enregistrés par la caméra, par le choix de la musique d'accompagnement (en se souciant du droit d'auteur), etc. 


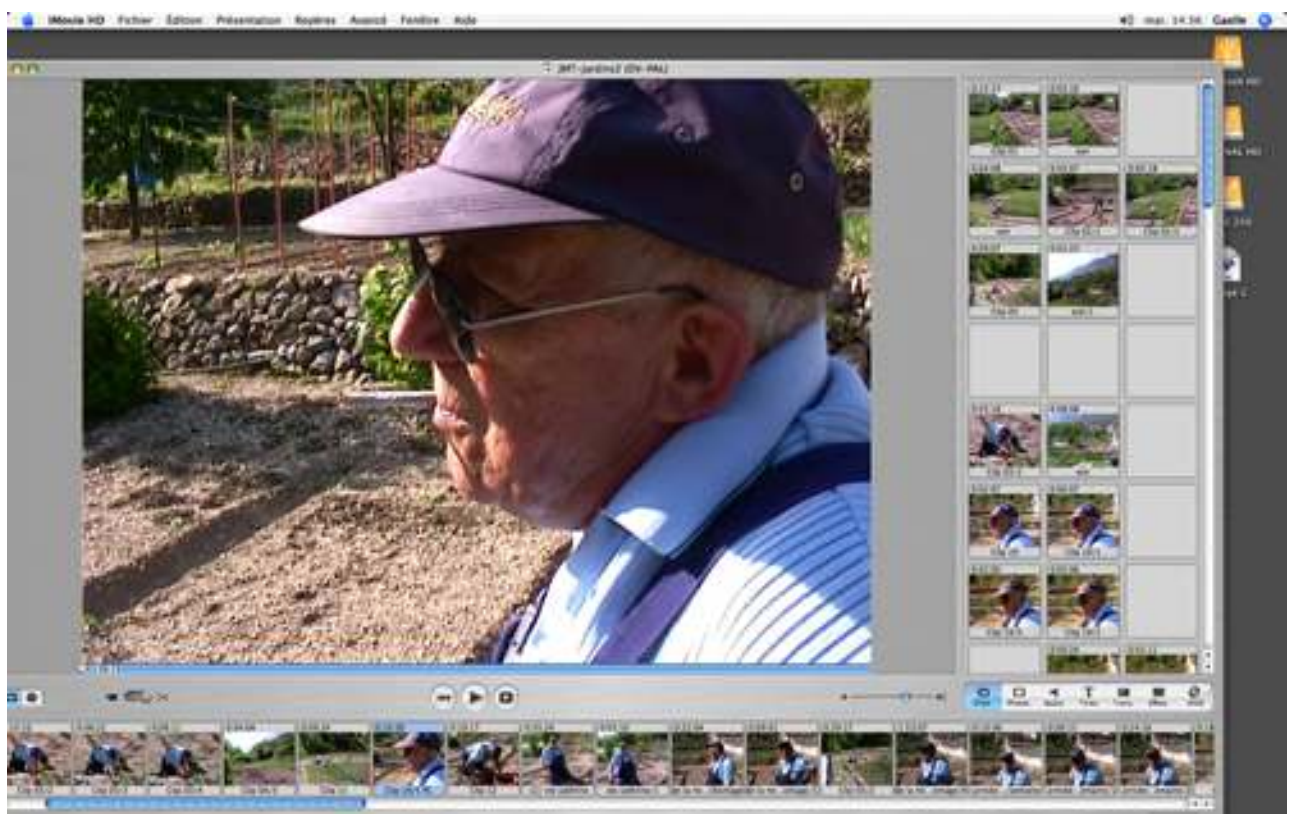

Dans iMovie, trois pistes de sons sont disponibles : sons réels, musique importée, voix off.

\section{Conclusion}

Les principaux liens institutionnels (et autres) avec l'extérieur sont les suivants :

-L'atelier géo-vidéo de Tolbiac (Université de Paris 1 Panthéon Sorbonne)

- CNRS Images

- CETSAH avec comme correspondant audiovisuel Marc-François Deligne

19 Une formation individuelle à l'écriture de synopsis et à la prise de vues est proposée à tous les membres de l'UMR. Le matériel est disponible pour les chercheurs de Prodig partant sur le terrain mais il est subordonné à une initiation courte à la prise de vues et de sons.

Les films documentaires ainsi réalisés peuvent être exploités par les enseignantschercheurs de diverses manières : comme produits autonomes à montrer dans le cadre d'un festival ${ }^{3}$, comme supports de cours, comme vidéos à intégrer à des projets multimédia combinant des photographies, du texte, des cartes, des graphiques, etc. et disponibles sur le web ainsi que sous forme de produits DVD. Par ailleurs, les films documentaires réalisés peuvent être édités sur support DVD par l'UMR PRODIG.

\section{NOTES}

1. X. Browaeys, « Géographie, image et vidéo. Pour une pratique de l'audiovisuel » in L'Information géographique, vol 63, 1999, pp. 25-32

2. L'UMR PRODIG dispose de 10000 photographies de plaques de verre à thème géographique, dont la plupart ont été numérisées.

3. Par exemple, le festival « Environnement et société », la « Biennale internationale du cinéma scientifique », le « festival international du film documentaire océanien », le 
festival « Territoire en Images », le festival «A nous de voir. Science et cinéma » d'Oullins ».

\section{RÉSUMÉS}

Cet article décrit l'activité audiovisuelle de l'UMR PRODIG en insistant sur l'importance de l'image en géographie. La méthodologie présentée aborde le travail du synopsis et du scénario avant de s'attacher à la prise de vues, au montage et au mixage. La conclusion replace la pratique vidéo dans le cadre plus large des projets multi-media.

This article describes the audiovisual activity in the PRODIG research unit and insists upon the importance of image in geography.

Considerations upon scenario and synopsis are presented through a methodology before considering shooting, cut and soundmixing. As a conclusion, the audiovisual practice is put back in the bigger plan of multi-media projects.

\section{INDEX}

Keywords : image, multimedia, audiovisual, geography, methodology

Mots-clés : audiovisuel, géographie, méthodologie

\section{AUTEUR}

\section{GAËLLE HALLAIR}

Gaëlle Hallair est Ingénieure d'études au CNRS UMR PRODIG [ghallair@univ-paris1.fr]. Elle est également doctorante sous la direction de Marie-Claire Robic Publication : Hallair Gaëlle, Le géographe Emmanuel de Martonne et l'Europe centrale, Grafigéo, Prodig (à paraître) 